\title{
The tenacious nature of memory binding for arousing negative items
}

\author{
DEANNa L. NovaK \\ University of California, Santa Cruz, California \\ AND \\ Mara Mather \\ University of Southern California, Los Angeles, California
}

\begin{abstract}
In two experiments, we investigated whether people are better or worse at updating memory for the location of emotional pictures than of neutral pictures. We measured participants' memories for the locations of both arousing negative pictures and neutral pictures while manipulating practice (encountering the same event repeatedly) and interference (encountering the same picture in a different location). Memory for the context of emotional items was less likely to be corrected when erroneous and was less likely to be correctly updated when the context changed. These results suggest that initial item-context binding is more tenacious for emotional items than for neutral items, even when such binding is incorrect.
\end{abstract}

Psychologists have long known that our ability to remember something over the long term depends on how we rehearse or reencounter that information (e.g., Bahrick, 1984; Ebbinghaus, 1885/1913; Lane, Mather, Villa, \& Morita, 2001; Marsh, 2007). Much research also indicates that memory differs between events that are emotionally arousing and events that are neutral (for a review, see Kensinger, 2008). However, the interaction between emotional arousal and repeated exposure or practice has received less attention (but see Mather, in press). Is emotional arousal likely to enhance or to hinder the updating of event representations in memory when events change? For instance, imagine that a shocking crime is initially described in the media with the name of a suspect but is updated later when the actual perpetrator is discovered. Will readers be less likely to remember the correct perpetrator later than if the crime were not so emotionally arousing?

Understanding how emotion affects memory updating is important. We often have repeated experiences that contribute to our memories about particular people, places, or events. Over time, external reality may change, but memories do not always adjust. For instance, errors in memory for prose passages can be surprisingly persistent, even when people have the opportunity to relearn the original information. For example, in one classic study (Kay, 1955), once a week for 6 weeks, participants tried to remember and write down a story that they had heard in their first experimental session. Immediately after each recall attempt, they heard the story again, giving them the opportunity to correct their memory for any inaccuracies the next time they recalled it. Participants rarely corrected their memories and instead repeatedly made the same recall errors, omissions, and additions. If the stories had been emotional, such as the description of a crime from various eyewitnesses, would memory correction have been more successful or less successful? In our study, we examined whether memory updating would be more likely or less likely for contextual details of arousing negative items than of neutral items.

People usually remember items that elicit emotional reactions better than they do items that do not spark an emotional response (e.g., Kensinger, Garoff-Eaton, \& Schacter, 2006; Mather \& Knight, 2005; Ochsner, 2000). However, emotional arousal does not lead to more accurate memory for all of an event's details. For instance, in one study (Christianson \& Loftus, 1991), participants watched a slideshow in which one slide depicted either a woman walking beside her bicycle or a woman lying wounded beside her bicycle. Participants who had seen the arousing version later had better memory for central details of the event, such as the color of the woman's coat, than did participants who had seen the neutral version. They also had poorer memory for peripheral details of the event, such as the color of a nearby car.

Accurate memory for events requires not only memory for individual items but also memory binding in order for one to remember the contextual details associated with various items and how the various items are related. Recent work has revealed that, in addition to having better item memory for emotionally arousing words or pictures, people also usually remember emotional items' colors or locations better than they do neutral items' colors or loca-

M. Mather, mara.mather@usc.edu 
tions (D'Argembeau \& Van der Linden, 2004; Doerksen \& Shimamura, 2001; Kensinger \& Corkin, 2003a, 2003b; Kensinger et al., 2006; MacKay \& Ahmetzanov, 2005; MacKay et al., 2004; Mather, Gorlick, \& Nesmith, 2009; Mather \& Nesmith, 2008).

Mather (2007) argued that the influence of arousal on memory binding depends on the relation of a to-beremembered feature to an arousing object. Mather's (2007) object-based framework suggests that, because emotional arousal enhances perceptual processing of arousing objects, it will tend to enhance the perceptual binding of features that are integral parts of those objects - that is, within-object features. On the other hand, arousal associated with objects will not enhance memory for betweenobject features of those objects - that is, features that are part of the same event but not of the emotional object. These initial benefits in perceptual binding will tend to lead to longer term benefits for memory binding, unless there is some intervening interference.

Of particular interest, recent studies (Mather et al., 2006; Mitchell, Mather, Johnson, Raye, \& Greene, 2006) have suggested that, when there is interference from previous associations, this will affect emotional associations more than will neutral associations, reversing the enhancing effects of emotional arousal on memory binding. For instance, participants have better memory for the location of arousing pictures than of neutral pictures when they are shown the pictures one at a time, with intervening tasks to prevent rehearsal (Mather et al., 2009; Mather \& Nesmith, 2008; Nashiro \& Mather, in press); but when given four picture-location pairings to rehearse in working memory, participants are worse at remembering the locations of pictures when all four pictures are emotionally arousing than when they are all neutral (Mather et al., 2006; Mitchell et al., 2006). This pattern suggests that emotionally arousing items either create more interference or are more disrupted by interference from other associations.

Findings from another study (Mather \& Knight, 2008) suggest that memory associations with arousing items lead to more memory interference for later related associations. Participants first learned that some harbinger cues (neutral tones or faces) predicted arousing negative or positive pictures, whereas others predicted neutral pictures. Then they were asked to learn associations between the harbinger cues and new contextual details, such as nearby digits and locations of the harbinger cues. Participants had worse memory for the associations between emotional harbingers and contextual details than for the associations between neutral harbingers and contextual details. This effect appeared to be due to proactive interference from previous associations with emotional items; new cues that were inherently emotionally arousing did not yield any memory impairments.

In the event that the configuration of our environment changes, we are dependent on the flexibility of our memory associations; they should be updated to represent the new relationships. For instance, in the example at the beginning of this article, a primary suspect of a crime may be resolved of guilt. In the event that we need to update memory, would it be more difficult for contextual details of an emotionally arousing experience than for those of a neutral experience? The finding that it is harder to learn new associations to emotional harbingers suggests that initial associations may be more likely to interfere with subsequent associations when the associated items are emotional than when they are neutral (Mather \& Knight, 2008).

To test this possibility, in the present study, we asked the participants to learn the locations of some emotional pictures and some neutral pictures. The paradigm we used was analogous to Kay's (1955) study, in which participants repeatedly made the same mistakes recalling a story, even when they were given the opportunity to update their memory associations by hearing the story again. In our study, the participants studied the locations of emotional and neutral pictures, attempted to recall the location of each picture, and then repeated the same study-test cycle multiple times. In Experiment 1, the study lists always had the same picture-location associations, and we compared rates of memory correction for neutral and emotional picture-location associations. In Experiment 2, some of the pictures changed locations after several study-test cycles, allowing us to compare memory-updating effectiveness for emotional picture-location associations with neutral picture-location associations.

\section{EXPERIMENT 1}

In Experiment 1, the participants learned the locations of neutral and emotionally arousing negative pictures by viewing the pictures in the same locations repeatedly. The participants completed a study block in which they viewed 64 pictures in one of eight locations on a computer screen, followed by a test block in which they were shown each picture and were asked to indicate its previous location. This study-test sequence was repeated until the participants had learned all of the picture-location associations. Location memory was measured across learning blocks for emotional and neutral pictures to determine how emotion would affect learning across multiple exposures to the information.

In the event that participants gave the wrong location for a picture during test, their tendency to correct the mistake on the next test was examined in order to determine whether memory updating would be affected by the emotion elicited by the picture. If emotional items were more affected by proactive interference from previous associations, participants should have found it more difficult to correct previous recall errors for the locations of negative arousing pictures, because initial erroneous location responses might have interfered with the creation of new, correct picture-location associations.

\section{Method}

Participants. Thirty-six undergraduate psychology students (23 female, 12 male, 1 declined to state) from the University of California, Santa Cruz, participant pool participated in this experiment to fulfill a course requirement. They ranged in age from 18 to 22 years $(M=18.81, S D=1.09)$.

Materials. Experiment 1 was designed with PsyScope software (Cohen, MacWhinney, Flatt, \& Provost, 1993) and administered with two Macintosh desktop computers: one for the experimenter 
and one for the participant. The stimuli were 64 matched-pair pictures assembled from sets that had been previously normed (Mather et al., 2009; Mather \& Nesmith, 2008); the stimuli also included some additional pairs. Each pair consisted of two pictures that had similar physical attributes and content but differed in arousal and valence. To confirm the differences between the emotional and neutral versions, we had 3 additional undergraduates rate all of the pictures (shown in random order) for arousal and valence on 9-point scales. The negative arousing pictures were rated as being much more arousing $(M=7.36, S E=0.14)$ than their matched neutral pictures $(M=$ $1.77, S E=0.11)[t(63)=34.51, p<.0001]$. As expected, the negative arousing pictures were also rated as being more negative $(M=$ $1.88, S E=0.10)$ than the neutral pictures $(M=5.61, S E=0.13)$ $[t(63)=-26.46, p<.0001]$. Furthermore, each negative arousing picture received a mean arousal rating that was higher than that of its neutral match and a mean valence rating that was lower than that of its neutral match.

Two sets of 64 pictures were created using the matched pairs. One set included the neutral version of Pairs 1-32 and the emotional version of Pairs 33-64; the second set included the emotional version of Pairs 1-32 and the neutral version of Pairs 33-64. The two sets were administered between participants; for example, when the first picture for one participant was an emotional picture in the bottom right-hand corner of the screen, a different participant saw its neutral pair as the first picture in that same location.

Picture locations formed a grid on the computer screen of three rows, each containing three locations. The center location was reserved for the presentation of pictures at test. The locations were randomly assigned to the pictures, with the constraint that each location displayed the same number of neutral and emotional pictures across the experiment. There were two sets of random location assignments, which led to a total of four stimuli lists when the two picture sets were crossed with the two location assignments.

Procedure. The participants were told that the experiment session would last up to $1 \mathrm{~h}$, depending on their performance. Since better performance would result in shorter sessions, the participants had an incentive to perform well. First, the participants completed a consent form and a demographic sheet. After completing the paperwork, the participants began the computer task. The participants completed the task at their own pace, but the experimenter remained in the room to answer questions if necessary. The task was to learn the locations of 64 pictures. The participants saw each of the 64 pictures for $3 \mathrm{sec}$ (ample time to attend to each picture and its location, but not enough time to rehearse more than a few associations) in a random order.

During the location recall test, each picture appeared in the center of the computer screen. A grid of the eight possible picture locations surrounded the picture, and each location was denoted by a number. The participants used the number keys of the keyboard to indicate the square in which they recalled the picture's having been located. After the randomly ordered 64 test trials, the experimenter checked the participants' answers. The experimenter was seated at the second desktop computer, which was networked to the participant's computer. This allowed the experimenter to locate the participant's data file remotely and check the participant's accuracy by copying and pasting the file into a Microsoft Excel spreadsheet that could tally mistakes. After each block, the experimenter told the participant whether all of the location test questions had been answered correctly. If any errors had been made, the participant had to complete another block, viewing the same pictures again in the same locations (in a different, random order) and then completing the recall test (again, in a different, random order). The session ended after the participant achieved the learning criterion of answering all of the location test questions correctly twice.

\section{Results}

We used an alpha level of .05 for all statistical tests and partial eta squared $\left(\eta_{\mathrm{p}}^{2}\right)$ as a measure of effect size.
All but 2 of the participants satisfied the learning criterion, recalling all 64 picture locations correctly, twice in the allotted $1 \mathrm{~h}$. Both of the participants who had not reached the criterion were stopped after seven iterations of viewing and testing, at which point they were very close to satisfying the criterion. One participant answered all of the recall questions correctly once but missed 1 picture location in his or her last 2 blocks; the other participant missed 1 picture location in each of his or her last 3 blocks. Their data are included in all analyses. It took the remainder of the participants between 4 and 12 blocks to learn the locations of the pictures $(M=6.34, S E=0.29)$.

A two-way repeated measures ANOVA including the within-subjects factors of block (with only the first four blocks included, since all of the participants completed at least four blocks) and picture type (emotional or neutral) revealed a main effect of block, indicating that participants were able to complete the task, and the number of location recall errors decreased significantly across blocks $\left[F(1.91,66.73)=480.25, p<.001, \eta_{\mathrm{p}}^{2}=.93\right]^{1}$ (see Table 1 for block means). There was also a main effect of picture type: Across learning blocks, participants made more location recall errors for emotional pictures $(M=6.29, S E=0.47)$ than for neutral pictures $(M=$ $5.42, S E=0.38)\left[F(1,35)=8.45, p<.01, \eta_{\mathrm{p}}^{2}=.19\right]$. However, a significant interaction revealed that the influence of emotion depended on block. During the first block, participants made slightly fewer location recall errors for emotional pictures $(M=14.50, S E=0.68)$ than for neutral pictures $(M=15.03, S E=0.79)$; but in subsequent blocks, participants made more recall errors for the locations of emotional pictures (Block 2, $M=6.28$, $S E=0.63$; Block 3, $M=2.94, S E=0.49$; Block 4, $M=$ $1.44, S E=0.38)$ than for the locations of neutral pictures (Block 2, $M=4.36, S E=0.54$; Block 3, $M=1.61, S E=$ 0.35 ; Block $4, M=0.67, S E=0.18)[F(1.65,57.62)=$ $4.84, p<.05, \eta_{\mathrm{p}}^{2}=.120$ ] (see Figure 1 ).

To better understand this interaction, we coded each mistake as either a repeat of a mistake made in an earlier testing block (e.g., if a participant had inaccurately responded "Location Number 7" for a picture that had actually appeared in Location Number 3, and then recalled "Location Number 7" again during a subsequent testing

Table 1 Mean Number of Pictures Recalled Incorrectly, by Block, for Each Picture Type in Experiment 1

\begin{tabular}{|c|c|c|c|c|c|}
\hline \multirow[b]{3}{*}{ Block } & \multirow[b]{3}{*}{$n$} & \multicolumn{4}{|c|}{ Picture Type } \\
\hline & & \multicolumn{2}{|c|}{ Neutral } & \multicolumn{2}{|c|}{ Emotional } \\
\hline & & $M$ & $S E$ & $M$ & $S E$ \\
\hline 1 & 36 & 15.03 & 0.80 & 14.50 & 0.68 \\
\hline 2 & 36 & 4.36 & 0.53 & 6.28 & 0.63 \\
\hline 3 & 36 & 1.61 & 0.35 & 2.94 & 0.50 \\
\hline 4 & 36 & 0.67 & 0.18 & 1.44 & 0.38 \\
\hline 5 & 31 & 0.39 & 0.16 & 0.74 & 0.23 \\
\hline 6 & 21 & 0.33 & 0.13 & 0.57 & 0.30 \\
\hline 7 & 17 & 0.24 & 0.18 & 0.24 & 0.18 \\
\hline
\end{tabular}

Note-There were 32 neutral pictures and 32 negative pictures. $n=$ number of participants who completed that block. 


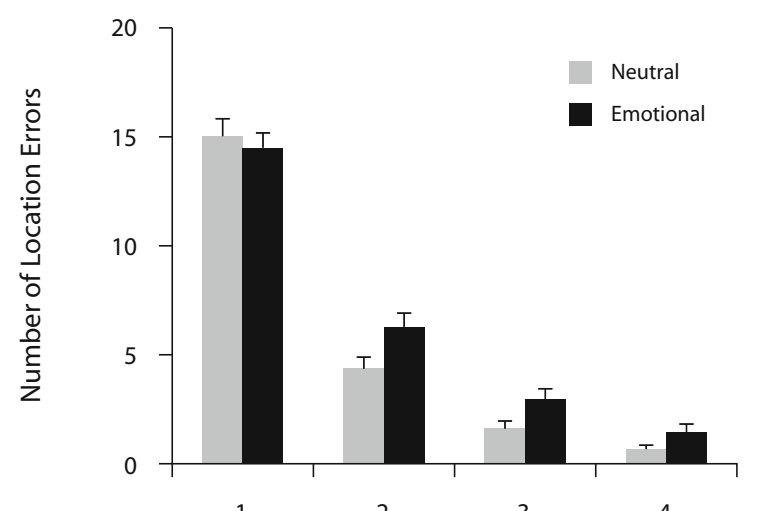

Block

Figure 1. Mean number of location recall errors for emotional and for neutral pictures, across four learning blocks. There was a significant arousal $\times$ block interaction where, initially, there was no significant difference in recall errors between emotional and neutral pictures; but in Blocks 2, 3, and 4, participants made more errors recalling emotional picture locations than recalling neutral picture locations.

block) or a new mistake and then computed the proportion of repeat mistakes for emotional and neutral items for each participant out of the total number of mistakes after Block 1. The mean proportion of repeat mistakes for locations of emotional pictures $(M=.42, S E=.03)$ was significantly greater than the mean proportion of repeat mistakes for locations of neutral pictures $(M=.29, S E=$ .03) $[t(32)=3.16, p<.01]$. One participant made no errors recalling negative image locations after the first block, and 2 participants made no errors recalling neutral image locations after the first block and had no potential to then make repeat errors for those types of images; therefore, they were omitted from this analysis.

We followed up this analysis with an item analysis, in which we computed the proportion of times that a particular item was responded to with the same error as on any previous trial out of the total number of times that that item was erroneously responded to (omitting Block 1 instances). Pictures that had higher average arousal ratings in our pretesting were more likely to elicit repeated location errors $[r(128)=.36, p<.001]$. Arousal and valence ratings were highly correlated $[r(128)=-.90, p<.001]$; thus, it is not surprising that repeated location errors were also correlated with valence ratings $[r(128)=-.31, p<$ $.001]$, so that more negative pictures were more likely to elicit repeated location errors.

\section{Discussion}

This study was designed to examine whether repeated learning and memory correction for picture-location associations would be more effective or less effective for emotional than for neutral stimuli. After seeing the pictures once, participants recalled about the same number of locations for emotional and neutral stimuli, but slightly more for emotional stimuli. Thus, baseline recall was close to equivalent for the two types of pictures in the first round of learning. This is different from the findings of previous research, which revealed an advantage for location memory for emotional stimuli. However, previous studies that found that arousal enhanced location memory typically used incidental encoding instructions (e.g., Mather et al., 2009; Mather \& Nesmith, 2008), whereas the nature of our study required intentional encoding instructions. Attention is one mechanism by which arousal benefits memory by focusing attention to certain stimuli. Intentional encoding strategies also lead to more focused attention and might minimize the deficit in attention to neutral stimuli, as compared with attention to emotional stimuli, when one is trying to learn the stimuli (see also D’Argembeau \& Van der Linden, 2004).

As predicted, an arousal-related difference in memory emerged after the first test block. By the second test block, participants' location memory for emotional pictures was worse than that for neutral pictures. The lag in learning the locations of emotional pictures, as compared with learning the locations of neutral pictures, was partially the result of repeating recall mistakes for emotional picture locations more frequently than those for neutral picture locations. This suggests that an initial incorrect association between an emotional picture and a location leads to more interference for learning the correct association than does an initial incorrect association for a neutral picture.

How could a location that the picture was never seen in interfere with learning the actual location? In cases in which a participant was not able to remember the location of a picture at first test, he or she may have attempted to aid recall by imagining that picture in some location, or he or she may have simply guessed the location. In either case, the inaccurate imagery or response may have led to a memory representation for an incorrect picture-location conjunction. In order to respond correctly on subsequent rounds, the participant would have needed to note the correct location in the next learning round and correct or update his or her memory. Our findings revealed that participants were less likely to make this correction when memories were emotional. In Experiment 2, we tested whether this impaired memory correction for emotional picture-location conjunctions could be due to greater proactive interference from previous associations for emotional items than from previous associations for neutral items.

\section{EXPERIMENT 2}

Experiment 2 followed the same format as that used in Experiment 1 and used the same stimuli; however, in Experiment 2, half of the pictures changed locations after the third learning block, and participants were asked to update their memory for that change. We compared the initial difference in location recall between emotional and neutral pictures (in the two blocks before the switch occurred) to the difference between the two types of recall after the switch (in the two blocks after the switch oc- 
curred). We predicted that proactive interference should be greater for emotional pictures than for neutral pictures, leading to poorer location recall for emotional pictures than for neutral pictures, after a switch.

\section{Method}

Participants. Twenty-seven undergraduate psychology students (5 male, 22 female) from the University of California, Santa Cruz, participant pool participated in this experiment to fulfill a course requirement. They ranged in age from 18 to 22 years $(M=18.74$, $S D=0.98)$

Materials. Experiment 2 used the same pictures as those used in Experiment 1. Experiment 2 also retained the two sets of random location assignments for each picture set. However, in Experiment 2, one location assignment was the original location of a picture, and the other location assignment was used for a location to which the picture could change (although only half of the pictures actually changed for each participant). Counterbalancing which half of the pictures from each list changed locations, as well as whether the emotional or the neutral version from a picture pair was shown, resulted in four stimulus lists.

Procedure. Experiment 2 used the same procedure as that used in Experiment 1, except that, in Experiment 2, we changed the location of half of the pictures after the third learning block, by which point - on the basis of the results from Experiment 1-we expected very high memory accuracy. The participants were told that, at some point during the session, some pictures might change locations, and that when that happened, they would be responsible for learning the new locations for the next test round.

As in Experiment 1, the participants were allowed to leave after they had reached a learning criterion; but because of the increased difficulty associated with pictures changing locations, the criterion in Experiment 2 was lowered, so that the participants would not perceive the task as being overwhelming. The learning criterion was to correctly answer all of the recall questions once (rather than twice, as in Experiment 1). If the participants had not met the criterion after having completed six blocks, they were allowed to leave and were dismissed. However, when they started, they were led to believe they would have to continue until they had satisfied the criterion.

\section{Results}

Of the 27 participants who completed this experiment, 9 were able to reach the learning criterion of correctly recalling all 64 picture locations once before reaching the fourth block, and so completed the experiment at that point. Since the change in picture locations occurred during the fourth block, their data could not be included in comparisons looking at recall before the change and after the change.

One initial question was whether participants would again show greater errors for emotionally arousing pictures in Blocks 2 and 3. Unlike in Experiment 1, the differences in errors for emotional and neutral items in this experiment were not significant in Blocks 2 and 3 (see Table 2 for block means). We also examined the proportions of repeated errors in Blocks 2 and 3. As in Experiment 1, a higher proportion of location errors was repeated from previous rounds for emotional pictures $(M=.31, S E=$ $.05)$ than for neutral pictures $(M=.19, S E=.03)[t(17)=$ $2.64, p<.05]$. Furthermore, item analyses for all trials from Blocks 1-3 and for the no-change trials from Blocks 4-6 revealed that the proportion of repeat errors across all no-location-change test opportunities for a particular item was correlated with its arousal rating $[r(128)=.22, p<$
Table 2

Mean Number of Pictures Recalled Incorrectly, by Testing Block, for Each Valence in Experiment 2

\begin{tabular}{|c|c|c|c|c|c|}
\hline \multirow[b]{3}{*}{ Block } & \multirow[b]{3}{*}{$n$} & \multicolumn{4}{|c|}{ Picture Valence } \\
\hline & & \multicolumn{2}{|c|}{ Neutral } & \multicolumn{2}{|c|}{ Emotional } \\
\hline & & $M$ & $S E$ & $M$ & $S E$ \\
\hline 1 & 27 & 13.11 & 0.88 & 13.48 & 0.80 \\
\hline 1 & 18 & 15.17 & 0.86 & 15.06 & 0.81 \\
\hline 2 & 27 & 3.81 & 0.65 & 4.00 & 0.51 \\
\hline 2 & 18 & 5.11 & 0.79 & 4.94 & 0.50 \\
\hline 3 & 24 & 1.25 & 0.24 & 1.58 & 0.37 \\
\hline 3 & 18 & 1.61 & 0.27 & 2.11 & 0.42 \\
\hline 4 (same location) & 18 & 1.11 & 0.40 & 1.39 & 0.38 \\
\hline 4 (changed location) & 18 & 5.22 & 0.55 & 6.72 & 0.60 \\
\hline 5 (same location) & 18 & 0.53 & 0.24 & 1.06 & 0.39 \\
\hline 5 (changed location) & 18 & 1.94 & 0.32 & 2.94 & 0.53 \\
\hline 6 (same location) & 14 & 0.36 & 0.23 & 0.50 & 0.36 \\
\hline 6 (changed location) & 14 & 0.36 & 0.17 & 0.93 & 0.53 \\
\hline
\end{tabular}

Note-There were 32 neutral pictures and 32 negative pictures. $n=$ number of participants who completed that block. Descriptive summaries in bold exclude participants who completed the experiment before the locations changed and who were not included in statistical analyses. Descriptive summaries in regular print include those participants who completed the experiment before the locations changed.

$.05]$, as well as with its valence rating $[r(128)=-.19$, $p<.05]$, replicating the results of Experiment 1. Thus, although there was no significant effect of emotion on the total number of errors in Blocks 2 and 3, emotion effects emerged when we examined the rate of repeat errors.

Our main focus of interest in Experiment 2 was the comparison of recall of picture-location conjunctions before and after we switched the locations. Thus, we compared recall for switched and nonswitched pictures from the two blocks before the location switch (Blocks 2 and 3) for each picture type with recall for switched and nonswitched pictures in the two blocks after the location switch (Blocks 4 and 5) for each picture type (see Table 2 for all block means). Then, to examine the influence of arousal on memory updating, phase (prior to and after the switch) and picture type (emotional or neutral) were included as factors in a two-way repeated measures ANOVA.

As expected, participants made more location recall errors after the switch $(M=10.28, S E=1.24)$ than before the switch $(M=6.89, S E=0.70)[F(1,17)=11.80, p<$ $\left..01, \eta_{\mathrm{p}}^{2}=.41\right]$. There was also a main effect of picture type, where, overall, participants made more recall errors for the locations of emotional pictures $(M=9.47, S E=$ 1.04) than for the locations of neutral pictures $(M=7.69$, $S E=0.85)\left[F(1,17)=5.77, p<.05, \eta_{\mathrm{p}}^{2}=.25\right]$. More interestingly, the interaction between picture type and phase was significant because of an increased difference in location recall errors between emotional and neutral pictures after the switch (for emotional pictures, $M=11.89, S E=$ 1.57; for neutral pictures, $M=8.67, S E=1.07)$, as compared with before the switch (for emotional pictures, $M=$ 7.06, $S E=0.77$; for neutral pictures, $M=6.72, S E=$ $0.92)\left[F(1,17)=4.53, p<.05, \eta_{\mathrm{p}}^{2}=.21\right]($ see Figure 2$)$.

We also analyzed the data from switched and nonswitched pictures separately (see Table 2 for means). For Block 4, a two-way repeated measures ANOVA with picture type (emotional or neutral) and location (changed or 


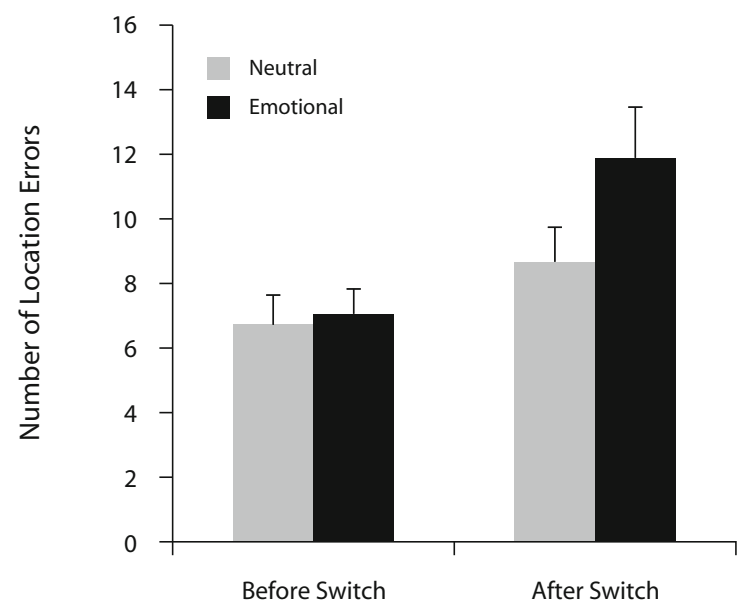

Figure 2. Mean number of location recall errors for emotional and for neutral pictures in the two blocks prior to half of the pictures changing locations and in the two blocks after the switch. There was a significant interaction where recall of the new locations of emotional pictures was worse after the switch than recall of the new locations of neutral pictures.

same) as factors revealed that participants made more errors for changed locations $(M=5.97, S E=0.52)$ than for constant locations $(M=1.25, S E=0.36)[F(1,17)=$ $\left.85.71, p<.001, \eta_{\mathrm{p}}^{2}=.83\right]$. In addition, participants made more errors for emotional pictures than for neutral pictures $\left[F(1,17)=7.38, p<.05, \eta_{\mathrm{p}}^{2}=.30\right]$. Furthermore, there was an interaction $\left[F(1,17)=5.93, p<.05, \eta_{\mathrm{p}}^{2}=\right.$ .26]. Follow-up $t$ tests revealed that the difference in errors between emotional and neutral picture locations was significant for changed locations $[t(17)=3.00, p<.01]$ but not for constant locations $[t(17)=0.93, p>.3]$. The same ANOVA repeated for Block 5 revealed a main effect of location constancy $\left[F(1,16)=35.34, p<.001, \eta_{\mathrm{p}}^{2}=\right.$ .69] and a main effect of emotion $[F(1,16)=4.96, p<$ $\left..05, \eta_{\mathrm{p}}^{2}=.24\right]$. Participants made more errors for emotional pictures $(M=2.00, S E=0.40)$ than for neutral pictures $(M=1.24, S E=0.20)$. However, there was not a significant interaction of the two factors $(F<1)$. By the fifth block, there were no significant effects. The fact that the emotion effects were strongest in Block 4 and declined in subsequent blocks is likely due to reduced power to detect differences as the number of errors decreased in Blocks 5 and 6.

These data support the hypothesis that emotional items are more subject to proactive interference and that this proactive interference makes it more difficult to update their features than it is to update neutral item features. However, there are alternative explanations for the increased difference between emotional and neutral picture location accuracy after the switch, as compared with before the switch. First, it is possible that the participants were less likely to notice that emotional pictures changed locations than they were to notice location changes of neutral pictures, and this might have led to poorer recall for emotional picture locations than for neutral picture loca- tions after the switch. For example, the negative pictures in this study may have been so upsetting that participants stopped attending to them after learning the original locations. To test this, a repeated measures ANOVA compared the proportion of mistakes after the switch (for pictures that changed) that were the result of responding with a picture's original location (which would suggest that participants were not aware that the picture had changed locations) and found that participants' mistakes were more likely to consist of the original location for neutral pictures $(M=.49, S E=.06)$ than for emotional pictures $(M=.28$, $S E=.05)\left[F(1,17)=8.58, p<.01, \eta_{\mathrm{p}}^{2}=.34\right]$. Thus, the increased failure in updating memory for emotional pictures cannot be attributed to less effective noticing of when emotional pictures changed locations than of when neutral pictures changed locations.

This finding - that when participants made postswitch location errors, they were more likely to respond with the initial location for neutral pictures than they were for emotional pictures - suggests a second possible account of the poorer postswitch location memory for emotional pictures. That is, that participants' initial picture-location learning was better for neutral than for emotional pictures, so their poorer recall of locations of emotional pictures after the switch was not the result of a failure at updating, but rather of increased confusion about the locations of those pictures after the switch because of poorer general memory for their locations. For example, in Experiment 1, in Blocks 2, 3, and 4, participants had poorer location recall for emotional pictures than for neutral pictures. Moreover, the last analysis found that participants were more likely to respond with the original location of a picture that had changed locations when the picture was neutral than when it was emotional. To control for initial learning, a repeated measures ANOVA compared the proportion of errors after the switch that involved a change in the location response, but only for those pictures for which participants had correct location recall in Blocks 2 and 3 (indicating that they had learned those pictures' original locations). This proportion was higher for emotional $(M=.46, S E=.05)$ than for neutral $(M=.29, S E=.05)$ pictures $[F(1,17)=4.51, p<$ $\left..05, \eta_{\mathrm{p}}^{2}=.21\right]$, indicating that participants often knew that emotional pictures had changed locations (as indicated by a change in which location they identified for a picture) but were not able to recall the correct new location.

This pattern is consistent with the hypothesis that memories of features of emotional objects are more susceptible to proactive interference than are memories for features of neutral objects. Even when participants had learned a picture's original location and noticed that it had changed its location, they had more difficulty forming or retrieving an accurate memory of its new location when the picture was emotional than when it was neutral. In contrast, participants showed no impairment in learning the locations of emotional pictures in the first block.

\section{Discussion}

Experiment 2 tested the hypothesis that memory updating is more difficult for emotional stimuli than for neutral 
stimuli, due to greater amounts of proactive interference. The experiment revealed that participants had greater difficulty updating their memory for changes to locations of emotional pictures than for changes to locations of neutral pictures. Follow-up analyses revealed that these failures in updating were not due to failures in noticing the changes in locations of emotional pictures. In fact, for pictures that changed locations, participants were more likely to respond incorrectly with the original location for neutral images than they were for emotional images. Furthermore, the impairment in updating locations for emotional pictures was not due to poorer memory for the original locations of emotional pictures than for neutral pictures; even for pictures that were equated for original learning, participants still made more updating errors for emotional images than for neutral images. These results support the hypothesis that emotional items are more affected by proactive interference from previous experience with the items, which hinders updating memory for new within-object features of objects.

One limitation of Experiment 2 is that participants who were able to learn the pictures fastest reached the learning criterion prior to the switch and could not be included in repeated measures analyses examining memory prior to and after the switch. Thus, the results can be generalized only to average and poorer performers. Since Experiment 1 also supports the hypothesis that initial bindings create more interference for subsequent learning for emotional objects than for neutral objects, and it did not omit data from the best performers, it is likely that the results from Experiment 2 will generalize to better performers. ${ }^{2}$

\section{GENERAL DISCUSSION}

The present study focused on the interaction between emotion and memory updating, in which a new association needs to be learned for some previously learned item. Two experiments revealed that memory updating is worse for emotional material, whether the updating needs to occur because of an initial misbinding or because of changes in external events. In Experiment 1, participants attempted to learn the locations of pictures across blocks in which they repeatedly saw the same pictures in the same locations. Initially, there was no difference in participants' recall for locations of emotional pictures than for neutral pictures. However, after the first block, participants made more recall mistakes for locations of emotional pictures than for locations of neutral pictures. The disparity in recall in later blocks between the two picture types was partially accounted for by the greater tendency to repeat recall mistakes for emotional pictures than for neutral pictures. Thus, errors were more tenacious for emotional items than for neutral items.

Experiment 2 tested the hypothesis that emotional memories are more difficult to update than neutral memories because they lead to more proactive interference. During three initial learning blocks, participants tried to learn each picture's location. They were then faced with a fourth learning block, in which half of the pictures had new locations, requiring memory updating. The experi- ment revealed that participants had more difficulty updating their memories for locations of emotional pictures than for locations of neutral pictures. This is consistent with the hypothesis that memory for the context of emotional items is more subject to interference from previous exposure to those items (Mather, in press).

Findings from another experimental paradigm (Mather \& Knight, 2008) also suggest that associations with emotional items lead to more interference for subsequent associations. In Mather and Knight's study, participants were exposed to harbinger cues, such as neutral tones or faces that either always preceded neutral pictures or always preceded emotional pictures. Then they were asked to learn associations between the harbinger cues and new contextual information, such as an associated digit or a location. Participants were worse at learning associations with emotional harbinger cues than they were at learning those with neutral harbinger cues. Thus, creating initial associations with emotional information seemed to lead to more interference for learning new associations than did creating initial associations with neutral information.

An important focus for future research would be to determine what characteristics of emotionally arousing negative pictures make it more difficult to update locations for them than for neutral pictures. Are the effects due to arousal or to valence? In the present study, all of the emotional pictures were negative, and the arousal and valence ratings for pictures were highly correlated. Thus, it is not surprising that both arousal and valence predicted the likelihood of failures of memory updating. In previous work that included both positive and negative pictures, we found that arousal predicted initial picture-location memory binding better than valence did (Mather \& Sutherland, 2009). Even more suggestive, in Mather and Knight's (2008) emotional harbinger study, participants were impaired at learning new associations to neutral items that had previously predicted positive pictures, and they were also impaired at learning new associations to neutral items that had previously predicted negative pictures. However, the relative contribution of arousal and valence to emotional interference effects needs further investigation.

In addition, the present study has examined memory updating for only one type of within-object feature: location. Thus, it is important to know whether the findings will generalize to other types of within-object features, as well as whether there might be differences in how memory updating for within-object versus between-item binding is affected by the emotional response elicited by the item (Mather, 2007).

Related to the issue of the type of association, the present study looked only at changes to memory requiring rebinding of the same type of feature. From these data, it is not clear whether proactive interference associated with binding for emotional objects may also hinder binding of a new feature that is different from those originally associated with the object. In the present study, participants learned the locations of pictures and had more difficulty updating their memory for the picture locations when the pictures were emotional than when they were neutral. Would participants also have a more difficult time 
binding a new feature, such as a colored border around an emotional picture, after having already learned the picture's location?

Research investigating the influence of arousal on memory has typically focused on isolated tests of general memory, without considering how memories might change. The present study contributes to this area of inquiry by examining differences between how the two types of memories adjust in the event that they are incorrect, or in order to remain consistent with a relationship that has changed in the environment. The results suggest that arousal associated with items leads to proactive interference that can prevent erroneous memories from being corrected and may also lead to difficulty updating memory when events change.

\section{AUTHOR NOTE}

This work was supported by NIA Grant AG025340. Correspondence concerning this article should be addressed to M. Mather, $3715 \mathrm{McClin}-$ tock Ave., University of Southern California, Los Angeles, CA 90089 (e-mail: mara.mather@usc.edu).

\section{REFERENCES}

BAHRICK, H. P. (1984). Semantic memory content in permastore: Fifty years of memory for Spanish learned in school. Journal of Experimental Psychology: General, 113, 1-29.

Christianson, S.-A., \& LofTuS, E. F. (1991). Remembering emotional events: The fate of detailed information. Cognition \& Emotion, 5, 81-108.

Cohen, J., MacWhinney, B., Flatt, M., \& Provost, J. (1993). PsyScope: An interactive graphic system for designing and controlling experiments in the psychology laboratory using Macintosh computers. Behavior Research Methods, Instruments, \& Computers, 25, 257-271.

D'Argembeau, A., \& Van der Linden, M. (2004). Influence of affective meaning on memory for contextual information. Emotion, 4, 173-188.

Doerksen, S., \& Shimamura, A. P. (2001). Source memory enhancement for emotional words. Emotion, 1, 5-11.

Ebbinghaus, H. (1913). Memory: A contribution to experimental psychology (H. A. Ruger \& C. E. Bussenius, Trans.). New York: Dover. (Original work published 1885)

KAY, H. (1955). Learning and retaining verbal material. British Journal of Psychology, 46, 81-100.

KENSINGER, E. A. (2008). Emotional memory across the adult lifespan. New York: Psychology Press.

Kensinger, E. A., \& CoRKIN, S. (2003a). Effect of divided attention on the memory benefit for negative as compared to neutral words. Brain \& Cognition, 51, 223-225.

Kensinger, E. A., \& Corkin, S. (2003b). Memory enhancement for emotional words: Are emotional words more vividly remembered than neutral words? Memory \& Cognition, 31, 1169-1180.

Kensinger, E. A., Garoff-Eaton, R. J., \& Schacter, D. L. (2006). Memory for specific visual details can be enhanced by negative arousing content. Journal of Memory \& Language, 54, 99-112.

Lane, S. M., Mather, M., Villa, D., \& Morita, S. K. (2001). How events are reviewed matters: Effects of varied focus on eyewitness suggestibility. Memory \& Cognition, 29, 940-947.
MacKay, D. G., \& AhmetZanov, M. V. (2005). Emotion, memory, and attention in the taboo Stroop paradigm: An experimental analogue of flashbulb memories. Psychological Science, 16, 25-32.

MacKay, D. G., Shafto, M., TaYlor, J. K., Marian, D. E., Abrams, L., \& DYER, J. R. (2004). Relations between emotion, memory, and attention: Evidence from taboo Stroop, lexical decision, and immediate memory tasks. Memory \& Cognition, 32, 474-488.

MARSH, E. J. (2007). Retelling is not the same as recalling: Implications for memory. Current Directions in Psychological Science, 16, 16-20.

Mather, M. (2007). Emotional arousal and memory binding: An objectbased framework. Perspectives on Psychological Science, 2, 33-52.

MATHER, M. (in press). When emotion intensifies memory interference. In B. H. Ross (Ed.), The psychology of learning and motivation (Vol. 51). Amsterdam: Elsevier. doi:10.1016/50079-7421(09)51003-1

Mather, M., Gorlick, M. A., \& Nesmith, K. (2009). The limits of arousal's memory-impairing effects on nearby information. American Journal of Psychology, 122, 349-370.

Mather, M., \& KNight, M. (2005). Goal-directed memory: The role of cognitive control in older adults' emotional memory. Psychology \& Aging, 20, 554-570.

MATHER, M., \& KNight, M. (2008). The emotional harbinger effect: Poor context memory for cues that previously predicted something arousing. Emotion, 8, 850-860.

Mather, M., Mitchell, K. J., Raye, C. L., Novak, D. L., Greene, E. J., \& Johnson, M. K. (2006). Emotional arousal can impair feature binding in working memory. Journal of Cognitive Neuroscience, 18, 614-625.

Mather, M., \& Nesmith, K. (2008). Arousal-enhanced location memory for pictures. Journal of Memory \& Language, 58, 449-464.

Mather, M., \& Sutherland, M. R. (2009). Disentangling the effects of arousal and valence on memory for intrinsic details. Emotion Review, 1, 118-119.

Mitchell, K. J., Mather, M., Johnson, M. K., Raye, C. L., \& Greene, E. J. (2006). A functional magnetic resonance imaging investigation of short-term source and item memory for negative pictures. NeuroReport, 17, 1543-1547.

NASHIRo, K., \& MATHER, M. (in press). How arousal affects younger and older adults' memory binding. Experimental Aging Research.

OCHSNER, K. N. (2000). Are affective events richly recollected or simply familiar? The experience and process of recognizing feelings past. Journal of Experimental Psychology: General, 129, 242-261.

\section{NOTES}

1. Data violated sphericity assumption, so we used the GreenhouseGeisser correction.

2. Furthermore, we checked the means in Experiment 1 for the proportion of repeat errors, and the best performers (those with zero or one error in Block 4) showed a slightly larger tendency to repeat errors for emotional $(M=.33, S E=.04)$ than for neutral $(M=.22, S E=.05)$ pictures than did those with more errors (for emotional pictures, $M=$ $.30, S E=.02$; for neutral pictures, $M=.25, S E=.03$ ). Furthermore, in Experiment 2, whereas the 18 lower performers did not have more errors for emotional than for neutral picture locations in Block 2, the 9 early completers had marginally higher errors for emotional picture locations $(M=2.11, S E=0.90)$ than for neutral picture locations $(M=1.22$, $S E=0.52)[t(8)=1.96, p<.09]$. Thus, if anything, there is some hint that the emotion effects were stronger for the high performers than for the low performers.

(Manuscript received September 10, 2008; revision accepted for publication May 10, 2009.) 